\title{
Diagnostic dilemma of Hodgkin's lymphoma versus tuberculosis: a case report and review of the literature
}

\author{
Anamika Banerjee ${ }^{\text {* }^{*}}$, Kaljit Bhuller ${ }^{2}$, Rajini Sudhir ${ }^{3}$ and Amrita Bajaj ${ }^{4}$
}

\begin{abstract}
Background: Hodgkin's Lymphoma $(\mathrm{HL})$ is a rare malignancy characterised histologically by the presence of ReedSternberg cells. Diagnosis of lymphomas can be difficult due to broad, non-specific presentations of disease, which can be similar to several other conditions ranging from infective, inflammatory or malignant causes, with one of the most common differentials being tuberculosis (TB). We aim to highlight the diagnostic dilemma of TB versus lymphoma with an atypical presentation of $\mathrm{HL}$ and explored areas for further research and improvement with a nonsystematic literature review using MEDLINE database and Google Scholar. Written consent was obtained from the patient in compliance with ethical guidelines.
\end{abstract}

Case presentation: A 23-year-old Asian female initially presented to rheumatology with over a one-year history of neuropathic pain, alongside abnormal white cell count and inflammatory markers. This was investigated with magnetic resonance imaging resulting in an incidental finding of mediastinal mass and pulmonary infiltrates. An initial diagnosis of TB was made despite testing negative for acid-fast bacilli and anti-tubercular treatment was commenced. Four months later, following clinical deterioration and further investigations, a mediastinal biopsy assisted in diagnosing Stage IV HL.

Conclusions: Lymphoma is often misdiagnosed as TB, prolonging time to treatment and may adversely impact patient prognosis due to disease progression. Existing TB guidelines for smear-negative cases are not clear when to consider alternative diagnoses. In smear-negative TB, lymphoma should be considered as a differential and definitive diagnostic tests such as molecular testing and histological examination of biopsies should be considered earlier in the diagnostic work-up to prevent diagnostic delay.

Keywords: Case report, Literature review, Hodgkin's lymphoma, Tuberculosis, Diagnostic delay, Guidelines

\section{Introduction}

Hodgkin's lymphoma (HL) is a rare B-cell lymphoma, accounting for $1 \%$ of all new tumour diagnoses worldwide. It is characterised by the presence of ReedSternberg cells and accounts for approximately 15\% of lymphomas (with $85 \%$ being non-Hodgkin) [1]. HL is more common in children and young adults, with the

\footnotetext{
*Correspondence: anamika.banerjee15@imperial.ac.uk

${ }^{1}$ Faculty of Medicine, Imperial College London, London, UK

Full list of author information is available at the end of the article
}

nodular sclerosis subtype of classical HL predominating in this age group [2].

Despite improved understanding of disease and advances in technology, lymphomas continue to present diagnostic challenges. The typical presentation of lymphomas includes palpable lymph nodes (most commonly in the neck) and B symptoms that is fever, night sweats and unexpected weight loss $>10 \%$ over the last 6 months [3]. However, lymphomas can have a wide variety of presentations especially in early-stage disease, resulting in diagnostic delay [4]. This applies to mediastinal and/ original author(s) and the source, provide a link to the Creative Commons licence, and indicate if changes were made. The images or other third party material in this article are included in the article's Creative Commons licence, unless indicated otherwise in a credit line to the material. If material is not included in the article's Creative Commons licence and your intended use is not permitted by statutory regulation or exceeds the permitted use, you will need to obtain permission directly from the copyright holder. To view a copy of this licence, visit http://creativecommons.org/licenses/by/4.0/. The Creative Commons Public Domain Dedication waiver (http://creativeco mmons.org/publicdomain/zero/1.0/) applies to the data made available in this article, unless otherwise stated in a credit line to the data. 
or pulmonary HL, which are often misdiagnosed. One of the most common differentials is tuberculosis (TB).

Various studies and case reports have highlighted the difficulties of distinguishing between lymphoma and TB [5], resulting in diagnostic delay which may worsen patient prognosis. This is still a problem today. Furthermore, TB and HL may coexist, which is confounding and brings further complexities.

We discuss a case of an atypical presentation of classical HL and literature review to raise awareness of this diagnostic dilemma and explore areas of further investigation and research to reduce diagnostic delay.

\section{Case presentation}

A 23-year-old Asian female presented to rheumatology in February 2020 following GP referral with history of worsening pain and paraesthesia of the right upper limb since 2018. She was managing with simple analgesics and physiotherapy under care of musculoskeletal and neurology team. Her symptoms were initially intermittent but became persistent from August 2019 and had progressed to involve the neck, upper-back and left arm. The severity of pain was disrupting her daily activities and sleep.

She was a non-smoker and non-drinker. There was no significant past medical history, except migraine.

She had a family history of Type 2 diabetes mellitus, hypertension and cancer, including thyroid and prostate cancer.

She was born in the UK to parents of Indian origin and had received the Bacillus Calmette-Guérin (BCG) vaccine at birth. She travelled to India every other year to visit relatives. She had no known contacts of TB.
Her weight was $54 \mathrm{~kg}$, and body mass index (BMI) was 21. On examination, there was slightly reduced power noted in the right upper limb and myofascial tenderness over the cervical and thoracic spine, but range of motion and reflexes remained fully intact.

Initial blood tests demonstrated elevated white cell count (WCC) of $14.8 \times 10^{9} / \mathrm{L}$ (normal range $(\mathrm{NR})$ $\left.4-11 \times 10^{9} / \mathrm{L}\right)$ with neutrophilia $\left(12.0 \times 10^{9} / \mathrm{L} ; \quad \mathrm{NR}\right.$ $\left.2-7.5 \times 10^{9} / \mathrm{L}\right)$, elevated C-reactive protein $(\mathrm{CRP})$ $(31.4 \mathrm{mg} / \mathrm{L} ; \mathrm{NR}<5 \mathrm{mg} / \mathrm{L})$, elevated total protein $(89 \mathrm{~g} / \mathrm{L}$; NR 60-83 g/L) with hypergammaglobulinaemia (49 g/L; NR 7-16 g/L), alongside low vitamin D (63.4 nmol/L; $\mathrm{NR}>70 \mathrm{nmol} / \mathrm{L}$ ). Auto-antibody screen including antinuclear antibody, rheumatoid factor and anti-citrullinated protein antibodies were negative.

Previous electromyography and nerve conduction studies in February 2019 reported no evidence of nerve lesions and no myopathic features at the time. In December 2019, neurologist arranged magnetic resonance imaging (MRI) of cervical spine, thoracic spine and brachial plexus which was initially reported as normal with respect to the spine and brachial plexus (Fig. 1). However, on further review of MRI at rheumatology multi-disciplinary team (MDT) meeting, an anterior mediastinal mass and lung shadowing was noted. Subsequent contrastenhanced computed tomography (CT) of neck, thorax, abdomen and pelvis (Fig. 2) demonstrated a cystic anterior mediastinal mass measuring $3.3 \times 3.8 \mathrm{~cm}$ thought to be a necrotic node which is typical of TB. There was evidence of consolidation in the right upper lobe with 'treein-bud' appearance. 'Tree-in-bud' refers to involvement of the smaller airways-a finding commonly seen in TB. The case was referred to chest MDT and was diagnosed
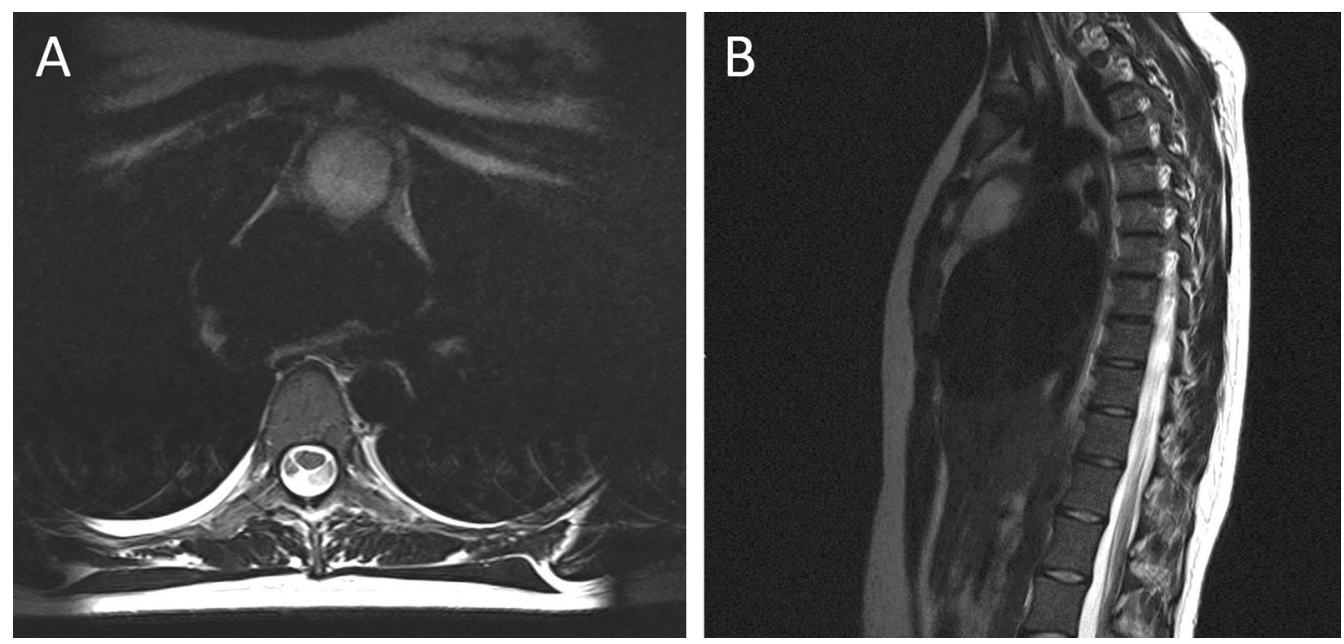

Fig. 1 Magnetic resonance imaging (MRI) demonstrating anterior mediastinal mass (A) Transverse T2 image showing a heterogeneous anterior mediastinal mass. B Sagittal fat suppressed image demonstrating anterior mediastinal mass 


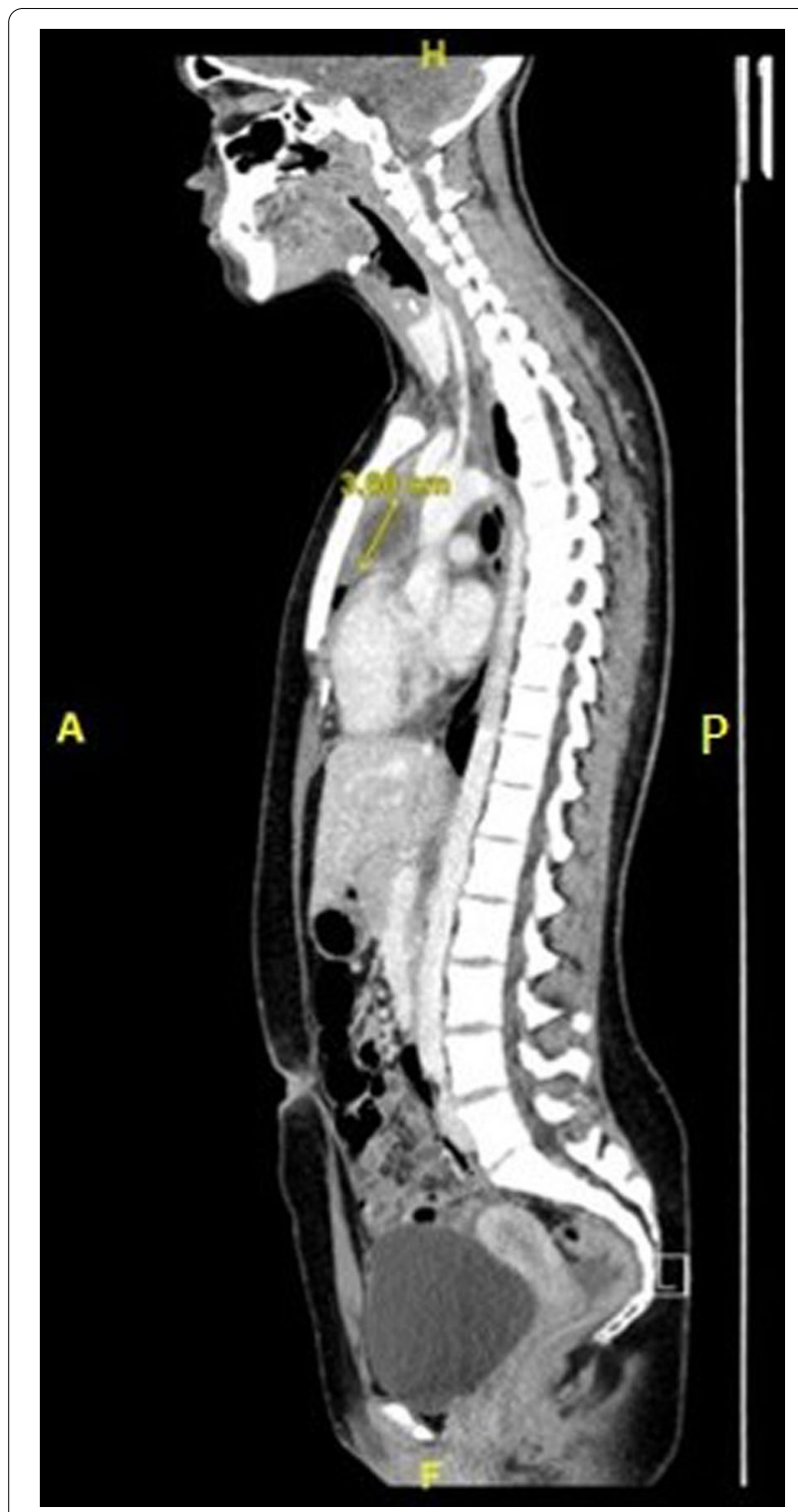

Fig. 2 Sagittal plane of CT. This image highlights the presence of a $3.3 \mathrm{~cm} \times 3.8 \mathrm{~cm}$ cystic mass in the anterior mediastinum

as likely pulmonary TB. However, in view of the clinicalradiological correlation, she did not have symptoms of cough, fever or chest pain at this time.

Although blood tests for enzyme-linked immune absorbent spot assay (ELISpot ${ }^{\circledR}$ ) were negative for TB and bronchoalveolar lavage (BAL) smear was negative for acid fast bacilli (AFB), she was treated empirically with the quadruple-antitubercular treatment Voractive ${ }^{\circledR}$ (combined tablet of rifampicin, isoniazid, pyrazinamide and ethambutol) with $10 \mathrm{mg}$ pyridoxine. The plan was to continue treatment and follow-up with repeat imaging in 2 months.
Within 2 weeks of commencing treatment, the woman developed continuous cough unable to sleep lying flat. She was advised to take simple cough linctus and continue with the treatment. However, her cough worsened, with progressive retrosternal discomfort.

Her care was transferred to a hospital closer to her home. The four-drug regimen was continued for 2 months followed by 1 month of Rifinah ${ }^{\circledR}$ (rifampicin and isoniazid) with pyridoxine $10 \mathrm{mg}$. Her cough and chest discomfort did not improve. Repeat blood tests performed in April and May 2020 showed haemoglobin fell from $117 \mathrm{~g} / \mathrm{L}$ to $101 \mathrm{~g} / \mathrm{L}$, WCC was persistently elevated at $25.1 \times 10^{9} / \mathrm{L}$ and $20.2 \times 10^{9} / \mathrm{L}$ with raised neutrophils of $22.49 \times 10^{9} / \mathrm{L}$ and $19.62 \times 10^{9} / \mathrm{L}$ respectively. Follow-up chest X-rays (CXR) and repeat CT scan demonstrated new cavitating lesions in the right lung with further enlargement of the mediastinal mass (Fig. 3). Sputum samples and repeat BAL sent for microscopy, culture and sensitivities (MC\&S) and polymerase chain reaction (PCR) with GeneXpert ${ }^{\circledR}$ testing again yielded negative results for TB. A CT-guided needle biopsy of the lung and fluid aspiration of the mediastinal mass was performed. This yielded necrotic tissue on histology with negative AFB smear, negative GeneXpert ${ }^{\circledR}$ and microbiology. Following this, a whole-body fluorodeoxyglucose-positron emission tomography (FDG-PET)-CT scan was performed demonstrating metabolically active necrotic lesions in the mediastinum and lung parenchyma (Fig. $4 \mathrm{~A}$ and $4 \mathrm{~B}$ ).

Histological analysis of mediastinotomy-derived lung biopsies demonstrated samples contained a nodular infiltrate of lymphoid cells admixed with bands of sclerotic tissue. The lymphoid tissue was polymorphous composed of small mature lymphoid cells admixed with histiocytes, eosinophils, neutrophils and some larger cells showing prominent nucleoli, binucleate forms and smudge cells. On immunohistochemistry, the large atypical cells were positive for CD30, CD15, MUM1, PAX5 and p53. They were negative for CD45, CD20, CD79a and CD3. The imaging and histological findings confirmed a diagnosis of Stage IVA classical Hodgkin's lymphoma (nodular sclerosing subtype) in June 2020.

TB treatment was discontinued and the patient was urgently referred to the haemato-oncology team and commenced on the escalated BEACOP-DAC regimen (bleomycin, etoposide, doxorubicin, cyclophosphamide, vincristine, prednisolone and dacarbazine, with granulocyte colony stimulating factor). As time was of the essence, the option of cryopreservation of ova prior commencing chemotherapy was not possible. However, gonadotrophin releasing hormone $(\mathrm{GnRH})$ analogue was offered. The FDG-PET-CT scan following the first two cycles demonstrated good response to chemotherapy 

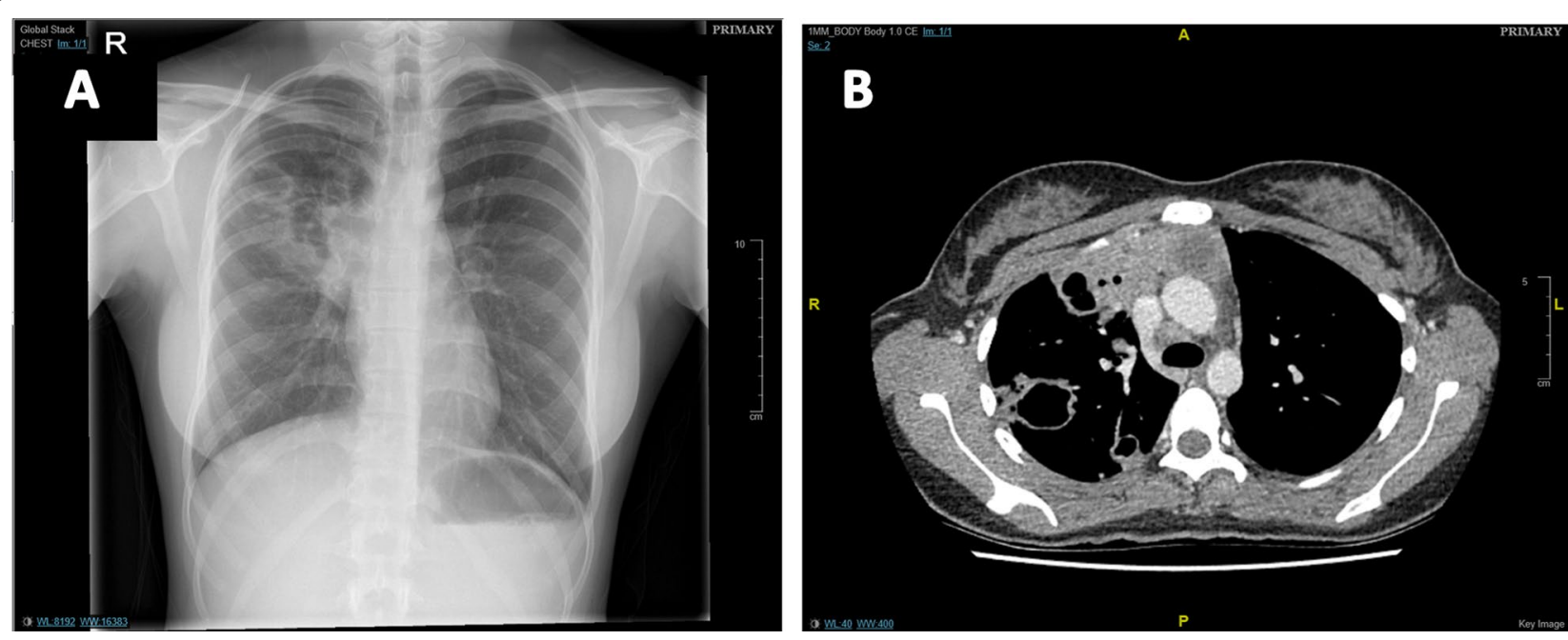

Fig. 3 Imaging demonstrating cavitating lung lesions. A Chest X-ray demonstrating cavitating lesions in the right upper lobe. B Contrast-enhanced Chest CT scan showing cavitating lesions in the right lung with multiple nodular lesions bilaterally, along with an anterior mediastinal mass that is continuous with the posterior sternum
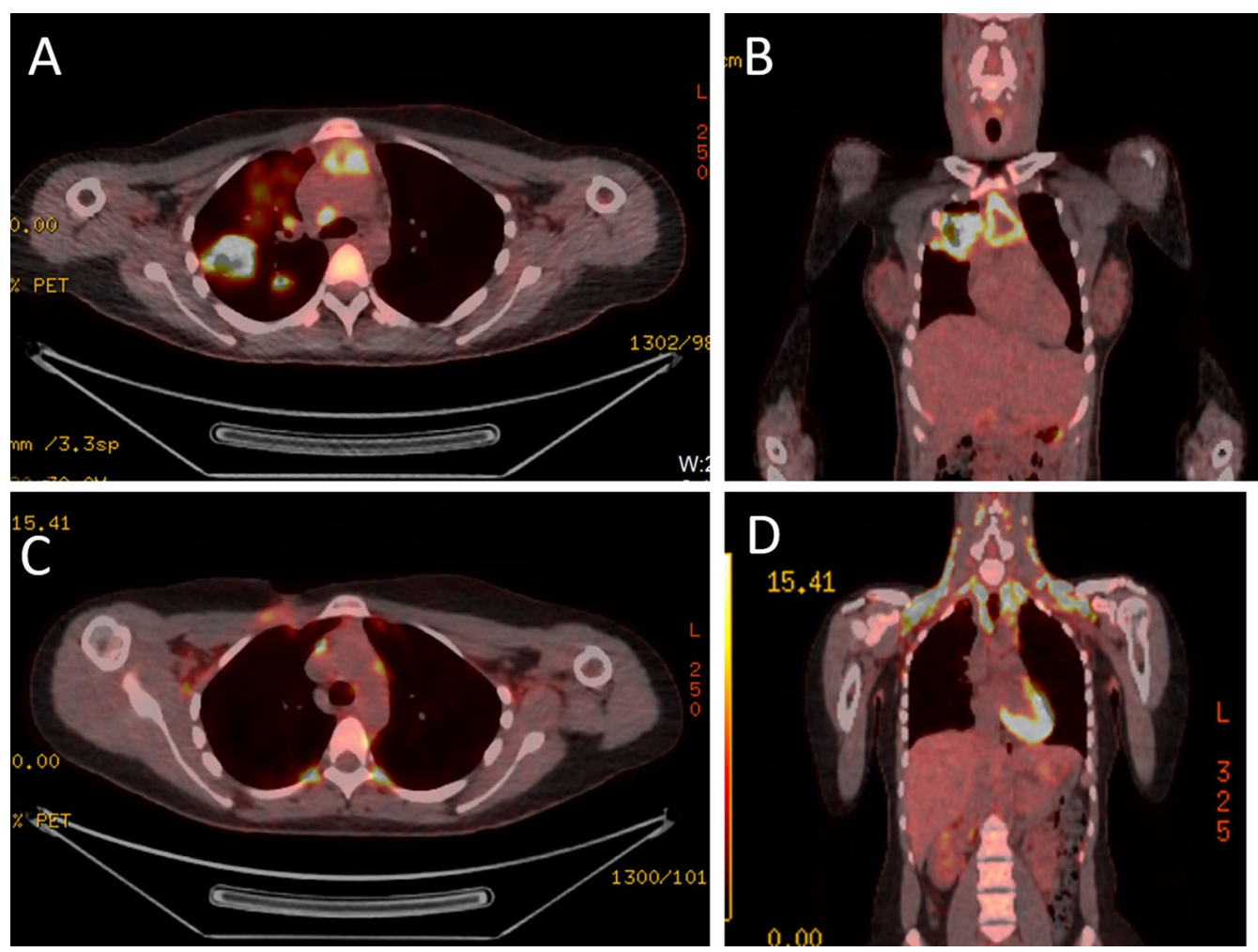

Fig. 4 FDG-PET-CT scan images pre-and post-chemotherapy. A Pre-treatment axial section of FDG-PET-CT at mid-thoracic level showing metabolically active necrotic mediastinal lymph nodes and parenchymal lesions in the lungs. B Pre-treatment coronal section demonstrating metabolically active necrotic mediastinal and lung lesions. C Post-treatment axial images of FDG-PET-CT at mid-thoracic level showing complete resolution of the previously noted lung lesions with no significant residual activity. Background uptake seen on these images is due to physiological brown fat activity. D Post-treatment coronal section of FDG-PET-CT with no residual metabolically active disease. Supraclavicular fossa shows marked background of physiological brown fat activity 
(Deauville Score 3). Therefore, only two more cycles of escalated BEACOP-DAC were given. The post-chemotherapy FDG-PET-CT scan (Fig. 4C and 4D) showed resolution of disease, so no further treatment was indicated. At the time of writing, the patient was well and plan was to follow-up in haemato-oncology clinic.

\section{Discussion}

As demonstrated in the patient's timeline (Fig. 5), the patient initially presented with possible neuropathic pain and was subsequently investigated with an MRI. Peripheral neuropathies can occur in $5-8 \%$ of patients with HL, which are mostly due to direct nerve compression by the tumour, deposition of immunoglobulins or secondary to treatment rather than true paraneoplastic phenomena [6]. However, paraneoplastic neuropathies can occur in B-cell lymphomas, the most common being GuillianBarre syndrome or chronic inflammatory demyelinating polyneuropathy (CIDP) [6]. In this case, as MRI findings did not reveal any neurological abnormalities, it is unlikely this occurred.

On further review of the MRI, mediastinal and pulmonary pathology were incidentally found and were confirmed by contrast-enhanced CT scan. The top differential based on clinical-radiological diagnosis was TB.

TB is one of the leading causes of mortality worldwide, affecting approximately 10 million people globally, with highest incidence in South East Asia and Africa [7]. In the western world, the UK is a key hotspot with over 5000 cases per year [8].
Pulmonary TB is divided into active and inactive forms. Imaging presentations of active TB include upper lobe consolidation with a 'tree-in-bud' appearance as well as lung cavitation [9], which was seen in this case. TB can also present with lymphadenopathy within in the mediastinum, which is typically necrotic giving it a cystic appearance on CT. This was believed to explain the cystic anterior mediastinal mass in this case. However, the first CT report also suggested the mediastinal lesion could be a benign thymic cyst or thymoma. Based on epidemiology, the patient's age and recent travel history, the diagnosis was in favour of TB and empirical treatment was commenced.

Where there is clinical suspicion of $\mathrm{TB}$, diagnosis typically involves imaging (usually CXR or CT) and confirmation with isolation of Mycobacterium tuberculosis from multiple samples of bodily fluids or tissue using AFB smear tests, cultures and/or rapid nucleic acid amplification tests for example GeneXpert ${ }^{\circledR}[9,10]$.

In this case, blood was tested for $\mathrm{TB}$ with ELIspot /T-SPOT.TB ${ }^{\circledR}$ blood test, and BAL smears were tested for $\mathrm{AFB}$, which were all negative for TB prior commencing treatment. TB culture results received 6 weeks later were also negative.

Investigations such as blood tests and sputum samples are not always able to identify the presence of TB. Swai et al. [11] demonstrated the typical diagnostic work-up for smear-negative TB used in clinical practice had poor sensitivity and specificity of $38.1 \%$ and $74.5 \%$ respectively. Therefore, due to the importance of controlling disease spread, current guidelines from the National Institute

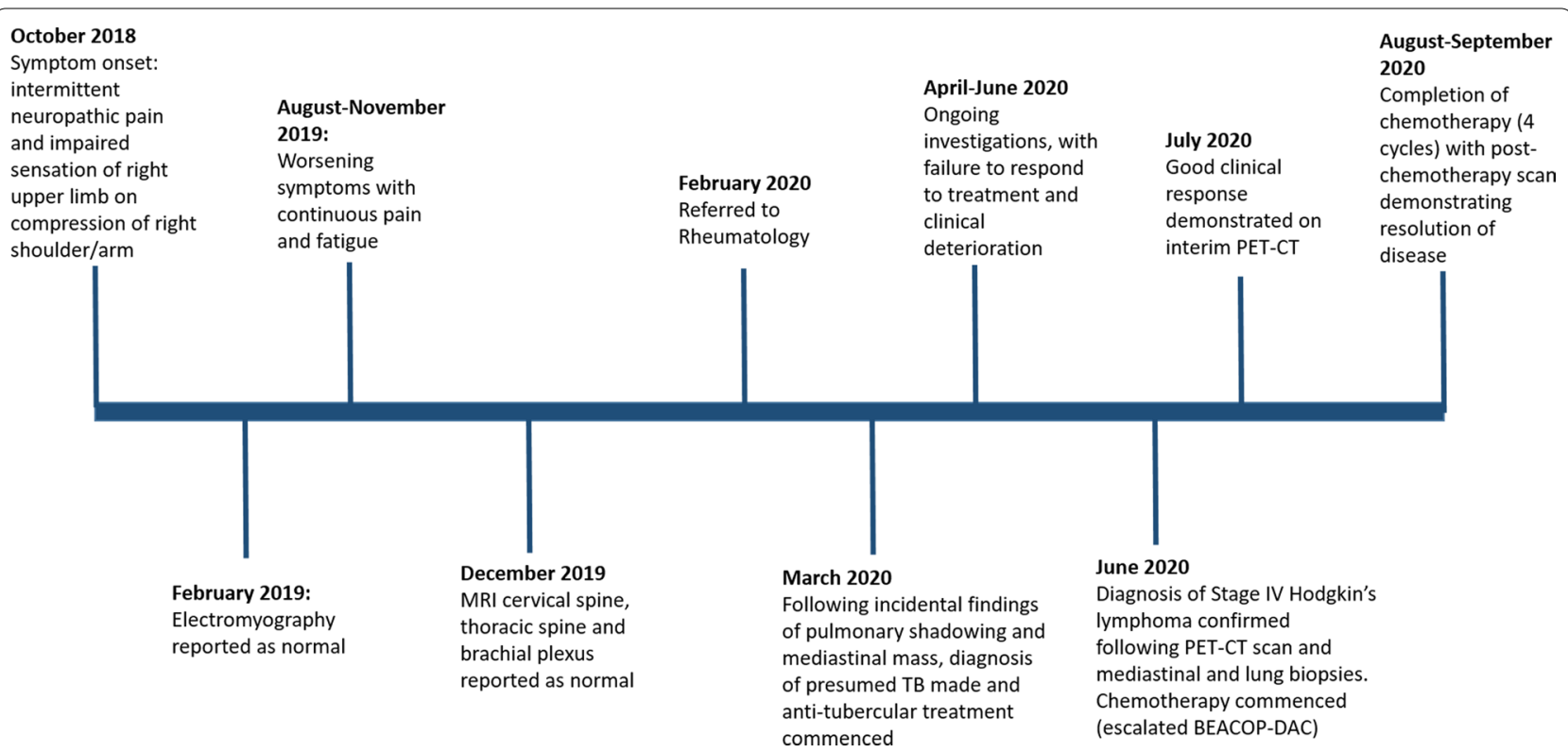

February 2019:

Electromyography

August-November

2019:

Worsening symptoms with continuous pain and fatigue

$\begin{array}{ll} & \\ & \begin{array}{l}\text { April-June 2020 } \\ \text { Ongoing } \\ \text { investigations, with } \\ \text { failure to respond }\end{array} \\ \text { February 2020 } & \text { to treatment and } \\ \text { Referred to } & \text { clinical } \\ \text { Rheumatology } & \text { deterioration }\end{array}$

July 2020
Good clinical
response
demonstrated on
interim PET-CT

August-September 2020

Completion of chemotherapy (4 cycles) with postchemotherapy scan demonstrating resolution of disease

\begin{abstract}
reported as normal
brachial plexus
\end{abstract} reported as normal

March 2020

Following incidental findings of pulmonary shadowing and mediastinal mass, diagnosis of presumed TB made and anti-tubercular treatment commenced

December 2019 MRI cervical spine,

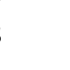

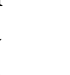

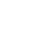


of Clinical Excellence (NICE) recommend if active TB is suspected, anti-tubercular therapy should be started "without waiting for culture results" and courses should be continued with close monitoring of response despite negative results if TB is still considered likely [10]. In this case there was no history of recurrent cough, chest pain and temperature on presentation.

Despite continuing anti-TB treatment, the patient's condition worsened. Repeated TB-focused tests were negative, and finally the diagnosis of HL was confirmed with an anterior mediastinal biopsy. We ask at what point should an alternative diagnosis be considered in a workup for TB and when should we consider performing a biopsy - the definitive diagnostic test which can identify both TB and lymphoma?

Current NICE guidelines for TB do not clearly specify at what stages should alternative diagnoses be considered, nor do they provide details of key distinguishing factors that could assist in differentiating these conditions $[10,12]$.

The challenging nature of diagnosing lymphomas has been previously reported. Both TB and lymphoma can have similar non-specific systemic symptoms (fever, weight loss and night sweats), as well as lymphadenopathy, and granulomatous inflammation on cytology and histology [13]. The non-specific symptoms can often make it difficult for patients to seek medical help or prompt appropriate referral from primary care to diagnose patients in early stages of disease [14].

Raising awareness of key differences between TB and lymphoma could help improve diagnostic accuracy in earlier stages of disease.

Features suggesting non-TB pathology include: no known exposure to TB or previous TB infection, involvement of supraclavicular lymph nodes, negative first-line TB investigations [for example tuberculin skin test (TST), sputum samples for AFB smears], and lack of response to anti-tubercular treatment within the first 2 months [13]. However, TST can give false positive results where BCG vaccine is given, as in this case. Therefore, Elispot ${ }^{\circledR}$ test (T-SPOT.TB ${ }^{\circledR}$ ) was performed.

A systematic review and meta-analysis demonstrated the T-SPOT.TB ${ }^{\circledR}$ test for blood samples had a greater pooled sensitivity of $81 \%$ (95\% CI $78-84 \%)$ for all patients with TB (confirmed and non-confirmed by culture) and 92\% (95\% CI 90-93\%) in those with culture-confirmed TB, compared to TST which only had sensitivity of $65 \%$ (95\% CI 61-68\%). However pooled specificity of the T-SPOT.TB ${ }^{\circledR}$ test was 59\% (95\% CI 56-62\%), whilst TST was $75 \%$ (95\% CI 72-78\%) [15]. Comparatively, the GeneXpert $^{\circledR}$ test using polymerase chain reaction (PCR) recommended by WHO and NICE have a sensitivity of $85 \%$ and specificity of $99 \%$ for detecting TB [16]. In our case, as the patient did not have symptoms of $\mathrm{TB}$ at presentation and ELIspot ${ }^{\circledR}$ and AFB smears of BAL were negative for TB, GeneXpert ${ }^{\circledR}$ should have been considered earlier to help rule out $\mathrm{TB}$.

Imaging plays an integral role in diagnosing $\mathrm{TB}$ and lymphoma and is usually performed early in the diagnostic work up. Key differences in the anatomical distribution of lymphadenopathy and enhancement patterns for TB and lymphoma in contrast-enhanced CT imaging could help improve diagnostic accuracy. Lymphoma has a heterogeneous imaging appearance depending on the subtype. Nodular sclerosing classical HL is common in young females and often presents as an anterior mediastinal mass as seen in this case [17]. Other features include (multiple) nodules or masses which can cavitate, as seen in our case. Tang et al. [18] identified mediastinal lymphadenopathy in TB typically presents with peri-hilar, peripherally enhancing lymph nodes with a cystic or necrotic centre, whereas lymphomas have a more central, homogenous enhancement and most commonly affects superior lymph node regions that is para-aortic lymph nodes. Further studies could help by identifying imaging appearances which can discriminate between the common differentials.

In cases of smear-negative $\mathrm{TB}$ or suspected drug-resistant TB, the definitive diagnostic tests, such as CT imaging, molecular testing that is GeneXpert ${ }^{\circledR}$ and (immuno-) histopathological examination of biopsies, should be performed much earlier in the diagnostic work-up, preventing delay [9]. Immunohistological diagnosis of classical HL typically demonstrates characteristic Reed-Sternberg/ Hodgkin cells positive for CD15 and CD30, with variable expression of CD20, but negative for CD79a, CD45 as seen in this case [19]. Although needle aspiration/biopsy is less invasive than excisional/incisional biopsy, several studies have demonstrated the sensitivity and specificity for diagnosing both TB and lymphoma are significantly higher with excision/incision biopsy, due to the larger sample and better quality of tissue obtained [15, 20]. For TB lymphadenitis, Knox et al. [21] found sensitivity of fine needle aspiration (FNA) specimens analysed via microscopy was $18 \%$, cytology was $38 \%$ and culture improved this to $85 \%$, but excision biopsy histology had highest sensitivity of $96 \%$.

For lymphoma, Hehn et al. [20] found $72 \%$ of lymphoma patients who had an FNA required subsequent excision biopsy to confirm diagnosis. The preferred sample is an excisional or incisional biopsy to obtain adequate tissue for analysis [3, 22]. This is particularly important for classical HL, as the characteristic Reed-Sternberg cells within the inflammatory milieu are often few and sparse and may be missed with FNA or core-needle biopsy as the reactive inflammatory 
Table 1 Lugano staging of classical Hodgyn lymphoma with 5-year net cancer survival rate

\begin{tabular}{|c|c|c|}
\hline $\begin{array}{l}\text { Lugano staging } \\
\text { classification }\end{array}$ & Definition & $\begin{array}{l}5 \text {-year net } \\
\text { cancer survival } \\
\text { rate (\%) }\end{array}$ \\
\hline । & $\begin{array}{l}\text { I-Single lymph node or one group of lymph nodes or one lymphatic organ affected } \\
\text { IE-one extra-nodal site in the absence of lymph-node involvement }\end{array}$ & 90 \\
\hline$\|$ & $\begin{array}{l}\text { II-Two or more groups of lymph nodes affected on the same side of diaphragam } \\
\text { IIE-Contiguous extra nodal extension from a nodal site with or without other groups of lymph } \\
\text { nodes on the same side of diaphragm (IIE) }\end{array}$ & 90 \\
\hline III & $\begin{array}{l}\text { Lymph nodes are affected on the same side of the diaphragam } \\
\text { III (1)-spleen/splenic nodes involved } \\
\text { III (2)-para-aortic, iliac, inguinal or mesenteric nodes involved }\end{array}$ & 80 \\
\hline IV & $\begin{array}{l}\text { Diffuse or disseminated disease with one or more extranodal sites affected including liver, lungs, } \\
\text { bone OR extra-nodal organs affected without associated lymph node involvement }\end{array}$ & 70 \\
\hline
\end{tabular}

Limited disease (Lugano Stage I and II) has the greatest prognosis and cure rates with 90\% 5-year survival, whilst advanced disease (Lugano Stage III and IV) has poorer prognosis, with 5 -year survival at $70-80 \%[3,22,26-28]$

background can make up to $99 \%$ of the cell population $[1,9,23]$. Specificities could also be improved by processing histological samples for whole genome sequencing and immunohistochemistry with flow cytometry.

Other obligatory investigations include full blood count, ESR, CRP, lactate dehydrogenase, liver function and viral screening for HIV, Hepatitis B and C [3]. Our patient's full blood count (FBC) showed elevated WCC, initially at $14.8 \times 10^{9} / \mathrm{L}$ in February rising to $25.1 \times 10^{9} / \mathrm{L}$ in April and remaining above $20 \times 10^{9} / \mathrm{L}$ prior commencing chemotherapy. In active TB infection, the WCC is usually within normal range and may rise to approximately $13.5 \times 10^{9} / \mathrm{L}$ [24]. Persistently elevated WCC above $15 \times 10^{9} / \mathrm{L}$ should raise concerns about lymphoma [25].

Findings from studies across the world highlight change is required to current guidelines to improve timing and accuracy of diagnosis of lymphoma. A study in South Africa investigating cases of lymphoma misdiagnosed as TB proposed a management algorithm where patients with presumed TB who are AFB smearnegative should initially be treated empirically with anti-tubercular medication, as per guidelines $[5,7,10]$. However, repeat testing and close monitoring must be implemented. If there is no improvement after 1 month of treatment and patient remains AFB smear-negative, then biopsies should be undertaken with histopathological analysis of TB as well as lymphoma and other possible pathology [5].

As shown in Table 1, although HL is very responsive to chemotherapy, it is an aggressive form of lymphoma and delay in diagnosis and treatment can affect prognosis [14]. Therefore, it is essential that a diagnostic approach involving earlier utilization of definitive tests described above as recommended by Puvaneswaran and Shoba [5] is implemented to update our guidelines for TB to prevent diagnostic delay of lymphoma.

\section{Conclusions}

Despite advances in knowledge, technology and medical practice, the diagnostic dilemma of TB and lymphoma still affects patients across the world. HL is a malignancy typically seen in children and young adults and should be considered as a differential in patients with lymphadenopathy. Imaging features can be difficult to differentiate from other presentations and correlation with clinical and biochemical findings is essential. Diagnostic difficulties can lead to increased number of investigations and delay in diagnosis and treatment of lymphoma and improve patient prognosis.

Existing clinical guidelines used in both primary care and in hospitals should be updated and refined to improve diagnosis at earlier stages of disease. In smearnegative $\mathrm{TB}$ cases, definitive diagnostic tests that is molecular testing with GeneXpert ${ }^{\circledR}$ and excisional/incisional biopsies could be considered earlier in the diagnostic work-up to distinguish between TB or lymphoma. Further research is required to develop better noninvasive diagnostic methods and better understand and address factors affecting diagnostic delay of lymphoma to improve patient care.

\section{Abbreviations}

AFB: Acid fast bacilli; BAL: Bronchoalveolar lavage; CT: Computed tomography; CXR: Chest X-ray; FDG-PET-CT: Fluorodeoxyglucose positron emission tomography-computed tomography; FNA: Fine needle aspiration; $\mathrm{HL}$ : Hodgkin's Lymphoma; MDT: Multidisciplinary team; MRI: Magnetic resonance imaging; TB: Tuberculosis.

\section{Acknowledgements}

We thank Dr Srikumar Mallick, Consultant in Respiratory Medicine, Kettering General Hospital NHS Foundation Trust for reviewing the article and for providing advice. 


\section{Authors' contributions}

Authors $A B, K B, R S$ and $A B$ contributed to the collection of information, patient care and writing of the manuscript. All authors read and approved the final manuscript.

\section{Funding}

None declared.

\section{Availability of data and materials Not applicable.}

\section{Declarations}

\section{Ethical approval and consent to participate}

Not applicable.

\section{Consent for publication}

Written informed consent was obtained from the patient for publication of this case report and any accompanying images. A copy of the written consent is available for review by the Editor-in-Chief of this journal.

\section{Competing interests}

The authors declare no competing of interests.

\section{Author details}

${ }^{1}$ Faculty of Medicine, Imperial College London, London, UK. ${ }^{2}$ Haematology, Children's and Adolescent Services, University Hospitals of Leicester NHS Trust, Leicester, UK. ${ }^{3}$ Respiratory Medicine, University Hospitals of Leicester NHS Trust, Leicester, UK. ${ }^{4}$ Radiology, University Hospitals of Leicester NHS Trust, Leicester, UK.

Received: 9 May 2021 Accepted: 24 May 2021

Published online: 19 July 2021

\section{References}

1. Agostinelli C, Pileri S. Pathobiology of hodgkin lymphoma. Mediterranean J Hematol Infect Dis. 2014;6(1):2014040. https://doi.org/10.4084/MJHID. 2014.040

2. Zhou L, Deng Y, Li N, et al. Global, regional, and national burden of Hodgkin lymphoma from 1990 to 2017: estimates from the $2017 \mathrm{Global}$ Burden of Disease study. J Hematol Oncol. 2019. https://doi.org/10.1186/ s13045-019-0799-1.

3. Eichenauer DA, Aleman BMP, Andre M, Federico M, Hutchings M, Illidge T, Ladetto M, ESMO Guidelines Committee. Hodgkin lymphoma: ESMO Clinical Practice Guidelines for diagnosis treatment and follow-up. HaematolMalig. 2018. https://doi.org/10.1093/annonc/mdy080.

4. Howell DA, Smith AG, Jack A, Patmore R, Macleod U, Mironska E, Roman E. Time-to-diagnosis and symptoms of myeloma, lymphomas and leukaemias: a report from the Haematological Malignancy Research Network. BMC Hematol. 2013. https://doi.org/10.1186/2052-1839-13-9.

5. Puvaneswaran B, Shoba B. Misdiagnosis of tuberculosis in patients with lymphoma. South African Med J. 2012;103(1):32-3. https://doi.org/10. 7196/SAMJ.6093.

6. Hagler KT, Lynch JW. Paraneoplastic Manifestations of Lymphoma. Clin Lymphoma. 2004;5(1):29-36. https://doi.org/10.3816/CLM.2004.n.007.

7. WHO (World Health Organisation). Tuberculosis. 2020. https://www.who. int/news-room/fact-sheets/detail/tuberculosis. Accessed 1 Oct 2020.

8. PHE (Public Health England). Tuberculosis in England: 2020 report (presenting data to end of 2019). 2020. https://assets.publishing.service.gov. uk/government/uploads/system/uploads/attachment_data/file/934954/ TB_Annual_Report_2020.pdf. Accessed 20 Oct 2020.

9. Ryu YJ. Diagnosis of pulmonary tuberculosis: recent advances and diagnostic algorithms. Tuberc Respir Dis (Seoul). 2015;78(2):64-71. https://doi. org/10.4046/trd.2015.78.2.64.

10. NICE (National Institute of Clinical Excellence). Tuberculosis-NICE guideline [NG33]. 2016. https://www.nice.org.uk/guidance/ng33. Accessed 30 Oct 2020.
11. Swai HF, Mugusi FM, Mbwambo JK. Sputum smear negative pulmonary tuberculosis: sensitivity and specificity of diagnostic algorithm. BMC Res Notes. 2011. https://doi.org/10.1186/1756-0500-4-475.

12. NICE (National Institute of Clinical Excellence). Non-Hodgkin's lymphoma: diagnosis and management. NICE guideline [NG52]. 2016. https://www. nice.org.uk/guidance/NG52. Accessed 30 Oct 2020.

13. Thakkar K, Ghaisas SM, Singh M. Lymphadenopathy: differentiation between tuberculosis and other non-tuberculosis causes like follicular Iymphoma. Front Public Health. 2016;4:31. https://doi.org/10.3389/fpubh. 2016.00031.

14. Thomson CS, Forman D. Cancer survival in England and the influence of early diagnosis: what can we learn from recent EUROCARE results? $\mathrm{Br} J$ Cancer. 2009;101(Suppl 2):102-9. https://doi.org/10.1038/sj.bjc.6605399.

15. Sester M, Sotgui G, Lange C, Giehl C, Girardi E, Migliori GB, et al. Interferon- $\gamma$ release assays for the diagnosis of active tuberculosis: a systematic review and meta-analysis. Euro Resp J. 2011;37(1):100-11. https:// doi.org/10.1183/09031936.00114810.

16. MacLean E, Kohli M, Weber SF, et al. Advances in molecular diagnosis of tuberculosis. J Clin Microbiol. 2020;58(10):e01582-19. https://doi.org/10. 1128/JCM.01582-19.

17. Tantisattamo E, Bello EF, Acoba JD. Nodular sclerosing Hodgkin's lymphoma presenting with a pseudo-breast mass extending from a necrotizing granulomatous mediastinal tumor. Hawaii J Med Public Health. 2012:71(8):212-7.

18. Tang SS, Yang ZG, Deng W, Shao H, Chen J, Wen LY. Differentiation between tuberculosis and lymphoma in mediastinal lymph nodes: evaluation with contrast-enhanced MDCT. Clin Rad. 2012;67(9):877-83. https:// doi.org/10.1016/j.crad.2012.02.006.

19. Mani H, Jaffe ES. Hodgkin lymphoma: an update on its biology with new insights into classification. Clin Lymphoma Myeloma. 2009;9(3):206-16. https://doi.org/10.3816/CLM.2009.n.042.

20. Hehn ST, Grogan TM, Miller TP. Utility of fine-needle aspiration as a diagnostic technique in lymphoma. J Clin Oncol. 2004;22(15):3046-52. https://doi.org/10.1200/JCO.2004.02.104.

21. Knox J, Lane G, Wong JSJ, Trevan PG, Karunajeewa H. Diagnosis of tuberculous lymphadenitis using fine needle aspiration biopsy. Int Med J. 2012;42(9):1029-36. https://doi.org/10.1111/j.1445-5994.2012.02748.x.

22. Cheson BD, Fisher RI, Barrington SF, Cavalli F, Schwartz LH, Zucca E, Lister TA. Recommendations for initial evaluation, staging, and response assessment of Hodgkin and non-Hodgkin lymphoma: the Lugano classification. J Clin Oncol. 2014;32(27):3059-68. https://doi.org/10.1200/JCO.2013.54. 8800.

23. Stein H, Delsol G, Pileri SA, Weiss L, Poppema S, Jaffe ES. Classical Hodgkin lymphoma. WHO CLassification of Tumours of Haematopoietic and Lymphoid Tissues. Lyon: IARC. 2008. p. 326.

24. Bregani ER, Valcarenghi C, Tien VT, Monzani V. Suggestive criteria for pulmonary tuberculosis in developing countries. Internat J Mycobacteriol. 2013;2(4):211-3. https://doi.org/10.1016/j.ijmyco.2013.08.004.

25. OlHI (The Oncology Institute of Hope and Innovation). Hodgkin's Lymphoma. https://theoncologyinstitute.com/diagnostics-list/hodgkinslymphoma/. 2020. Accessed 10 Nov 2020.

26. Follows GA, Ardeshna KM, Barrington SF, Culligan DJ, Hoskin PJ, Linch D, Sadullah S, Williams MV, Wimperis JZ, The British Committee for Standards in Haematology. Guidelines for the first line management of classical Hodgkin lymphoma. British J Haematol. 2014. https://doi.org/10.1111/ bjh.12878.

27. Hoffbrand V, Moss P, Pettit J. Essential Haematology, 5th edn. Wiley. 2006. Accessed 10 Nov 2020.

28. ONS (Office of National Statistics). 2019. Cancer survival by stage at diagnosis for England 2013-2017 edition of data set. https://www.ons.gov.uk/ peoplepopulationandcommunity/healthandsocialcare/conditionsanddi seases/datasets/cancersurvivalratescancersurvivalinenglandadultsdi agnosed. Accessed 10 Nov 2020.

\section{Publisher's Note}

Springer Nature remains neutral with regard to jurisdictional claims in published maps and institutional affiliations. 